\title{
Verzeichnis der Bearbeiter der 9. Auflage
}

Erwin Abele, Rechtsanwalt in München

Dr. Horst Baumann, emeritierter Professor an der Technischen Universität Berlin

Dr. Rola7nd Michael Beckmann, Professor an der Universität des Saarlandes

Dr. Oliver Brand, LL.M. (Cambridge), Professor an der Universität Mannheim

Dr. Alexander Bruns, LL.M. (Duke Univ.), Professor an der Albert-Ludwigs-Universität Freiburg

Dr. Christoph Brömmelmeyer, Professor an der Europa-Universität Viadrina, Frankfurt (Oder)

Dr. Heinrich Dörner, Professor an der Westfälischen Wilhelms-Universität Münster

Dr. Jan Dreyer, Rechtsanwalt und Fachanwalt für Versicherungsrecht in Hamburg

Charlotte Echarti, Rechtsanwältin in Rellingen

Dr. Jan Eichhorn, Rechtsanwalt in Hamburg

Dr. Thomas Gädtke, Rechtsanwalt in München und Lehrbeauftragter an der Ludwig-Maximilians Universität München

Dr. Sven Gerhard, Euler Hermes AG, Hamburg

Dr. Maximilian Guth, LL.M. (Southampton), Rechtsanwalt in Hamburg, Solicitor of England \& Wales

Dr. Olaf Hartenstein, D.E.A. (Sorbonne), LL.M. (Assas), Rechtsanwalt in Hamburg

Dr. Helmut Heiss, LL.M. (Chicago), Professor an der Universität Zürich

Dr. Jörg Henzler, Rechtsanwalt in Stuttgart

Dr. Harald Herrmann, emeritierter Professor an der Friedrich-Alexander-Universität ErlangenNürnberg

Dr. Knut Höra, Notar, Rechtsanwalt und Fachanwalt für Versicherungsrecht in Frankfurt am Main

Dr. Detlev A. Huber, Rechtsanwalt in Freiburg i.Br.

Jens Jaeger, Rechtsanwalt in Hamburg, Lehrbeauftragter an der Universität Hamburg

Dr. Katharina Johannsen, Vorsitzende Richterin am Hanseatischen OLG a.D., Hamburg

Dr. Ralf Johannsen ( $\dagger$ ), Rechtsanwalt in Hamburg

Dr. Rocco Jula, Rechtsanwalt und Fachanwalt für Versicherungsrecht in Berlin

Dr. Kai-Oliver Knops, Professor an der Universität Hamburg

Dr. Robert Koch, LL.M. (McGill), Professor an der Universität Hamburg

Dr. Hubertus W. Labes, Vorstandsvorsitzender der Hamburger Internationale Rückversicherung AG, Lehrbeauftragter an der Universität Hamburg

Dr. Kent Leverenz, Richter am LG Hamburg

Dr. Annemarie Matusche-Beckmann, Professor an der Universität des Saarlandes, Saarbrücken

Dr. Helmut Müller, Präsident des Bundesaufsichtsamts für das Versicherungswesen a.D., Berlin

Dr. Ernst Niederleithinger, Ministerialdirektor beim Bundesministerium der Justiz a.D., Honorarprofessor der Ruhr-Universität Bochum, Berlin

Dr. Peter Präve, Syndikus beim GDV, Berlin, Lehrbeauftragter an der Freien Universität zu Berlin

Jürgen Raab, Rechtsanwalt in Hamburg

Dr. Reinhard Renger, Ministerialrat beim Bundesministerium der Justiz a.D., Bonn

Dr. Jens-Berghe Riemer, Rechtsanwalt und Fachanwalt für Versicherungsrecht sowie Transportund Speditionsrecht in Nürnberg

Dr. Claus von Rintelen, Rechtsanwalt und Fachanwalt für Versicherungsrecht in Hamburg

Dr. Christian Rolfs, Professor an der Universität zu Köln

Dr. Christian Schneider, Rechtsanwalt in Köln 
Dr. Winfried Schnepp, Rechtsanwalt und Fachanwalt für Versicherungsrecht in Köln

Arno Schubach, Rechtsanwalt und Fachanwalt für Versicherungsrecht in Frankfurt am Main Dr. Dieter Schwampe, Honorarprofessor an der Universität Hamburg, Rechtsanwalt in Hamburg

Dr. Hans-Peter Schwintowski, Professor an der Humboldt-Universität zu Berlin

Dr. Ansgar Staudinger, Professor an der Universität Bielefeld

Dr. Wolfgang Voit, Professor an der Philipps-Universität Marburg

Dr. Eckhardt Wilkens, Vorstand der R+V Versicherung AG und Vorsitzender des Vorstandes der Vereinigten Tierversicherung Gesellschaft auf Gegenseitigkeit a.D., Burgwedel

Dr. Gerrit Winter, emeritierter Professor an der Universität Hamburg 